\title{
Contention-Aware Admission Control for Ad Hoc Networks
}

\author{
Yaling Yang \\ University of Illinois at Urbana-Champaign \\ yyang8@uiuc.edu
}

\author{
Robin Kravets \\ University of Illinois at Urbana-Champaign \\ rhk@cs.uiuc.edu
}

\begin{abstract}
An admission control algorithm must coordinate between flows in order to provide guarantees about how the medium is shared. In wired networks, nodes can monitor the medium to see how much bandwidth is being used. However, in ad hoc networks, communication from one node may consume the bandwidth of neighboring nodes. Therefore, the bandwidth consumption of flows and the available resources to a node are not local concepts, but related to the neighboring nodes in carriersensing range. Current solutions do not address how to perform admission control in such an environment so that the admitted flows in the network will not exceed network capacity. In this paper, we present a scalable and efficient admission control framework - Contention-aware Admission Control Protocol (CACP) - to support QoS in ad hoc networks. We demonstrate the effectiveness of CACP with both mathematical analysis and simulation results.
\end{abstract}

\section{Keywords}

Admission control, ad hoc network, Quality of Service, contention aware, ad hoc routing

\section{INTRODUCTION}

The expanded availability of small wireless computers has enabled the design and deployment of mobile ad hoc networks. Such networks provide the benefit of ease of deployment and independence on any preexisting infrastructure. Many suggested applications for these networks include multimedia data that require quality of service (QoS) support to provide effective communication. While many solutions for QoS support have been proposed in wired networks, the differences between wired and wireless communication demand the design of new solutions for ad hoc networks. Since the nodes in an ad hoc network must cooperate with each other to provide routing, they must also cooperate with each other to provide QoS support. Such cooperation includes policing at the endpoints of the flows and admission control along the routes to prevent new flows from consuming too many resources and disrupting the guarantees made to existing flows. Assuming the support of well-policed flows, the goal of our research is to provide an effective, scalable admission control protocol for wireless ad hoc networks so that end-to-end connections with QoS requirements can be established and maintained.

The goal of any QoS support is to provide applications with guarantees in terms of bandwidth, delay or jitter. Providing such guarantees in a networked environment requires support from multiple layers in the protocol stack. In particular, resource allocation at individual nodes must consider resources at the MAC layer, while route establishment must consider resources along the entire route of communication. While many approaches have dealt with such QoS support in wired networks, the physical characteristics of wireless networks do not lend themselves well to such guarantees. The main problem stems from the way that wireless nodes share the medium. Essentially, nodes that cannot communicate with each other directly may still contend directly with each other for the same resources. This affects resource allocation at individual nodes, since allocation decisions will require communication with nodes outside of its communication range, and along the entire route, since contention for resources may involve multiple nodes along a route. Our research provides admission control based on knowledge of these characteristics of wireless communication.

In this paper, we present CACP (Contention-aware Admission Control Protocol), which provides admission control for flows in an ad hoc network based on knowledge of local resources at a node as well as knowledge of the effect of admitting the new flow on neighboring nodes. Currently, CACP focuses on QoS support in terms of bandwidth allocation, however simulations show that by controlling bandwidth allocation, delay and jitter can also be controlled. The novelty of CACP comes from the fact that neighboring nodes are not limited to nodes in communication range but also include nodes in interference range (i.e., carrier-sensing range). This expansion of the definition of neighbor brings complexity in terms of communication overhead. We demonstrate through mathematical analysis and simulations that CACP is effective in terms of overhead, while maintaining effective use of the available communication resources.

The rest of the paper is organized as follows: Section II presents the characteristics of wireless communication relevant to admission control in ad hoc networks. Section III discusses the challenges of providing admission control in ad hoc networks. 
Section IV describes how CACP solves these challenges in detail. Section V provides analysis and simulation results of CACP. Finally, Section VI concludes our work.

\section{Characteristics of Wireless COMmunicAtion}

Allocation of communication resources is always necessary for communication over a shared medium. In a wired network, communication resources are well-defined. A node knows that whatever resources it uses will be directly removed from the pool of resources available to the other nodes sharing the medium. Additionally, all nodes see the same amount of physical resources available to the pool. In a wireless network, this is no longer true. Essentially, the characteristics of the shared wireless medium do not provide a unified view of the medium to all nodes. This difference comes from two physical differences between wired and wireless communication as described in detail in the following subsection. The problem is increased when considered in the context of an ad hoc network with dynamically moving mobile nodes.

\section{A. Wireless Channels}

The first difference between wired and wireless networks lies in the openness of wireless communication channels. Whether point-to-point or shared, wired links can be isolated to ensure that only authorized devices can use the communication channel. Wireless links are inherently shared and no such isolation is possible. Any node with a wireless transmitter can simply send data and contend for the wireless channel. There is also no isolation from other sources that may be using the channel with a completely different infrastructure (e.g. IEEE 802.11 and Bluetooth) or simply causing noise (e.g. a microwave oven). While we certainly make no claim to provide a solution for such interference, our approach dynamically monitors the communication medium to base resource allocation and admission control decisions on the current state of the wireless channel.

The second difference also stems from the physical characteristics of wireless communication. The structures of point-topoint and shared medium wired networks share the characteristic that nodes can hear each other's transmission and see the same channel state. However, for a wireless ad hoc network, the channel no longer maps to a physical medium, but instead maps to a physical space. Therefore, each node has a different view of the state of the communication channel. Consider the example in Figure 1. Communication from Node A impacts both Node B, which is inside Node A's transmission range, and Node C, which is outside Node A's transmission range, but inside its carrier-sensing range (typically, carrier-sensing range is around twice transmission range). Node A may know that it is sharing the channel with Node C, but it can not communicate directly with Node C since it can not decode Node C's packets correctly, and so does not know Node C's identity. Also, although Node A knows that it is sharing the channel with Node B, it does not know what other nodes are also sharing with Node B if they are outside Node A's carrier-sensing range (e.g., Node D in Figure 1). Although Nodes A and B know that they share the channel with each other, they do not observe the same channel state. Node A knows of Node B and senses Node C, implying that for Node A, the only nodes sharing the channel are Nodes A, B and the unknown C. Node B, on the other hand, knows of Nodes A and C and senses Node D. Therefore, Node B sees a channel shared by different nodes than Node A.

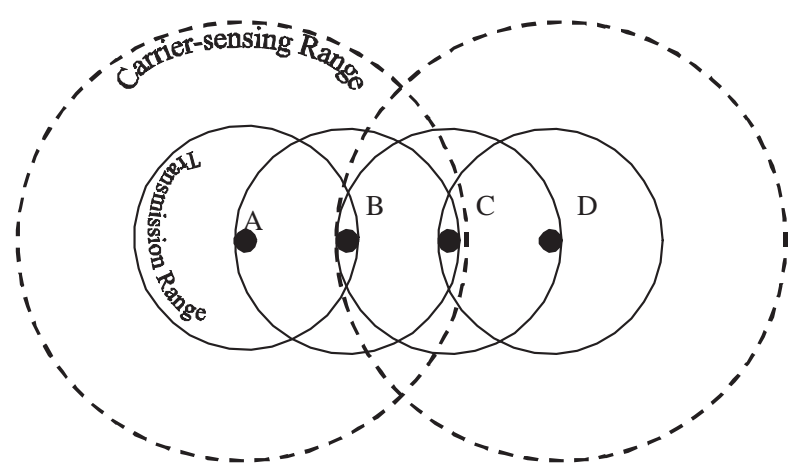

Figure 1. Contention Between Nodes

This unique structure of wireless channel introduces three challenges.

- Prediction of Available Bandwidth: In shared medium wireless networks, when a node starts to transmit a flow, it consumes bandwidth at its neighbors. Because each node has a different view of the network, the node cannot decide on its own whether its neighbors have enough bandwidth for the new flow.

- Contacting Relevant Neighbors: Contacting neighbor nodes is not easy, because a node may consume the bandwidth of a neighbor, but not be able to directly communicate with that neighbor if the neighbor is located outside transmission range and inside carrier-sensing range. 
- Prediction of Flow Bandwidth Consumption: Because multiple nodes on a route may contend for bandwidth at a single location and not know about each other, a node on the route of a flow cannot tell how much bandwidth the flow will consume at its neighbor nodes.

In Section III, we discuss these challenges in detail and briefly introduce how CACP addresses them.

\section{B. Mobility}

While it may be possible to find a feasible route for a flow, there is no guarantee of the stability of the route or that those resources will remain available. Route instability is caused by the mobility of nodes, which is expected to result in short-lived links between nodes as the nodes move in and out of range of each other. Mobility will also have an impact on available bandwidth since available bandwidth may decrease as communicating nodes move into range of each other. During the active period of a connection, a topological change may break a route or a commitment of service. The period of re-establishment of the route can take a long time if the network is partitioned or there is no route that has enough bandwidth. Hence, strict QoS, as in wired networks, cannot be guaranteed in an ad hoc network when mobility is present. We support the idea introduced in [1] that states that QoS requirements in ad hoc networks should be relaxed to allow a better-than-best-effort service. The QoS commitment that CACP provides is that no node is going to intentionally break the QoS commitments to the flows in the network by admitting too many flows. However, when the commitment is broken due to mobility, a notification message can be sent to the source indicating changes in the route. The source can either search for a new route or reduce its QoS requirement to accommodate a broken or degraded route.

Since there is no isolation between communicating nodes in ad hoc networks, the management of communication resources depends on knowledge of communication in the network and network topology. It is challenging to find a solution that balances the cost of collecting information with the usefulness of that data when mobility is present. This is particularly important when dealing with admission control. To provide the ideal solution, complete knowledge of the network is needed at the point where and when decisions are being made. Any delay in collecting that information may result in ineffective QoS management. The challenge comes from the fact that the mobility in ad hoc networks generates a tension between the benefits of accurate information and the costs of acquiring that information. Essentially, the limited bandwidth of wireless ad hoc networks leads to the need to limit any overhead from control information in the network. Additionally, in a mobile ad hoc network, information gathered about the network will have a limited lifetime since node mobility may change available resources and topology. Therefore, it is best to collect information as close as possible to the time that it is needed. Unfortunately, if information is collected too late, the network behavior may become unpredictable. To address mobility, CACP coordinates admission control in an on-demand fashion and so ties message overhead to the presence of requesting flows. CACP further limits message overhead by filtering out routes during an initial route discovery phase. In CACP, admission control is only performed once for flows as they request admission. Since mobility may break routes after a QoS commitment has been made to a flow, it will be necessary for the routing algorithm and the admission control protocol to either adapt to a patched route or start from scratch and find a new route. Currently, CACP falls back on the second approach, though we are exploring new techniques to reduce the need to perform a completely new admission request.

\section{Existing Approaches}

As discussed in previous subsections, the differences and challenges in wireless mobile ad hoc networks demand a very different perspective on network QoS management. In current research, TDMA-based approaches are proposed to provide QoS in wireless ad hoc networks [2][3][4][5][6]. However, such approaches require effective synchronization between all nodes in the network. Applying highly synchronized solutions in an ad hoc network becomes expensive and synchronization can fail when the nodes are mobile. The slot allocation algorithm in TDMA schemes is also vulnerable to mobility in the network since slot allocations must be reconfigured whenever there are changes in available bandwidth or changes to routes in the network.

As a result, single channel MAC layer scheduling approaches have been suggested to provide resource allocation in ad hoc networks [7][8][9]. In these approaches, only one channel is shared between all nodes. QoS is realized by coordinating the transmission schedules of packets between nodes. For example, in [8] and [9], the priority of a node's head-of-line packet is piggybacked onto control and data packets. Each node adjusts its backoff time according to the priorities of all its neighbor flows to achieve priority-based scheduling. In [7], nodes publish random schedules by exchanging seeds. When the schedules of several nodes collide, a probability that is related to the QoS requirement is used to determine which node can transmit. Although these approaches are based on localized decisions and, hence, are more flexible in the presence of mobility, they all focus on the packet level and only deal with resource allocation at individual nodes. They do not address how admission control can be performed along an entire route so that resources on the whole route can satisfy QoS requirements while at the same time not deplete the resources assigned to existing flows. 
Recently, some solutions related to admission control in ad hoc networks have been proposed [1][10][11][12][13][14]. However, work such as INSIGNIA [11], MMWN [13] and connectionless routing architecture [14] are very high level so that no consideration is given to the detailed differences between wired and wireless networks. Others, like SWAN [10], VMAC [12] and Monarch [1], do not give enough attention to the fact that when making admission control decisions, a node must not only consider local resources, but also consider resources of its neighbors since it may consume their resources through contention.

Our work fills this hole through the use of a framework for admission control in ad hoc networks called Contentionaware Admission Control Protocol (CACP). In CACP, when making admission control decisions, a node considers both local resources and resources at its neighbors. The challenge to CACP lies in the fact that information about contention cannot as easily be obtained as in a wired network. Since each node can only observe the amount of its local resources, neighbor resource information must be collected. Such collection will impose additional overhead and more contention on the network. Our research addresses these challenges with the goal of providing an effective, scalable admission control protocol for wireless ad hoc networks so that end-to-end connections with QoS requirements can be established.

\section{Challenges of Admission Control in AD-HOC Networks}

The aim of admission control is to determine whether the available resources in a system can meet the requirements of a new flow while maintaining bandwidth levels for existing flows. As described in Section II-A, the challenges of achieving this goal in ad hoc networks come from the characteristics of the wireless channel and mainly affect three issues: predicting the available bandwidth in the network, contacting relevant neighbor nodes and predicting the bandwidth consumption of a flow. In this section, we discuss these challenges and their possible solutions.

\section{A. Prediction of Available Bandwidth}

The first challenge to CACP is evaluating the available bandwidth in the network, so that the bandwidth requirements of all the flows do not exceed the resources in the network. Since each node sees a different channel state, the available bandwidth in the network is not simply a local concept. Two terms need to be defined: neighborhood available bandwidth and local available bandwidth. Neighborhood available bandwidth is the maximum amount of bandwidth that a node can use for transmitting without depriving the reserved bandwidth of any existing flows in its carrier-sensing range. Local available bandwidth is the amount of unconsumed bandwidth as observed by a given node. Since a node can consume bandwidth of nodes that are in its carrier-sensing range, the relationship between these two terms is that the neighborhood available bandwidth for a given node is equal to the smallest local available bandwidth of all the nodes in its carrier-sensing range. To successfully admit a flow, a node must have enough local and neighborhood bandwidth.

To demonstrate this, we show a simple simulation. In this scenario, there are six mobile hosts configured as shown in Figure 2.The radio transmission range is 250 meters and the carrier-sensing range is 550 meters. Node A is out of Node C's transmission range, but within its carrier-sensing range. Node E is in Node A's transmission range and is out of Node C's carrier-sensing range. Three $750 \mathrm{Kbps}$ CBR flows with packet size of 1000 bytes are established between Nodes E and F, Nodes A and B and Nodes C and D. At 12 seconds, Node A initiates Flow 1 to Node B. At 22 seconds, Node E initiates Flow 2 to Node F. Finally, at 32 seconds, Node C initiates Flow 3 to Node D. The bandwidth of the wireless channel is 2 Mbps. Figure 3 shows the changes in local available bandwidth at each source node as the three flows start successively. Figure 4 shows the effect on the throughput and delay for each flow over time.

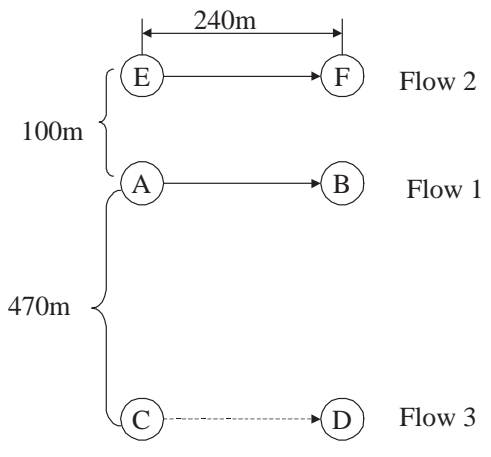

Figure 2. Simulation Topology to Demonstrate Neighborhood and Local Available Bandwidth

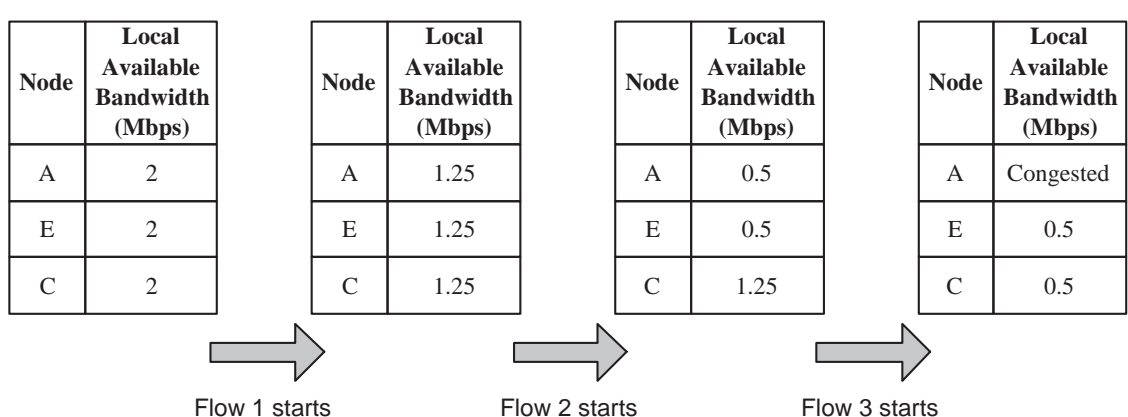

Figure 3. Changes of Local Available Bandwidth 


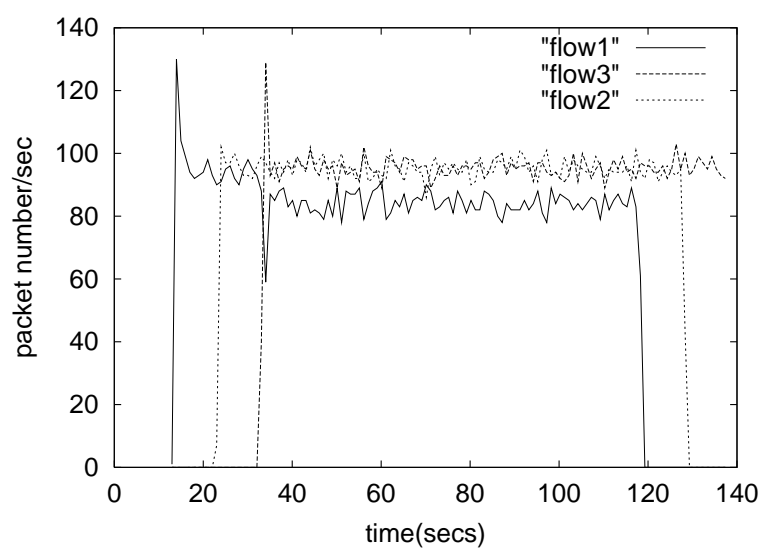

(a) Throughput of Flow 1 to 3

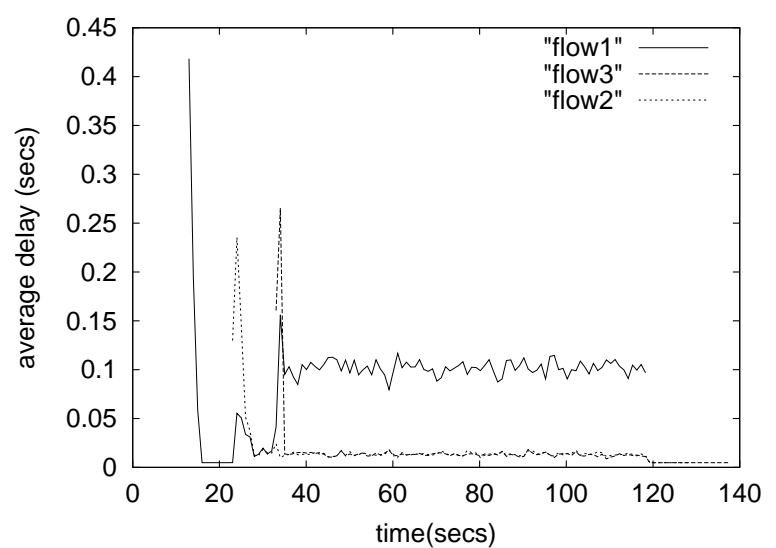

(b) Delay of Flow 1 to 3

Figure 4. Throughput and delay of Flow 1, 2 and 3

As shown in Figure 3, after Flows 1 and 2 start, Node C has $1.25 \mathrm{Mbps}$ local available bandwidth that is not consumed by the contention from Flow 1. Therefore, to Node C, there is enough local available bandwidth to admit Flow 3 . Therefore, when Flow 3 starts, it can get its desired throughput and delay as shown in Figure 4. In previous admission control approaches [1][10][12], since no consideration is given to neighborhood available bandwidth, Node C would admit Flow 3 . However, since Node A is in Node C's carrier-sensing range, Flow 3 consumes Node A's local available bandwidth. As shown in Figure 4, when Node C starts Flow 3, the contention from Flow 3 actually decreases the throughput of Flow 1 by $10 \%$ and increases the delay of Flow 1 dramatically. The reason for the decrease of QoS to Flow 1 is that Node A only has 0.5 Mbps local available bandwidth before Flow 3 starts, which is not enough for Flow 3. In other words, Node C does not have enough neighborhood available bandwidth for Flow 3. Therefore, enough local available bandwidth is not sufficient to accept a flow. There must also be enough neighborhood available bandwidth.

Since local available bandwidth is defined as the unconsumed bandwidth at a given node, each node can determine its own local available bandwidth by passively monitoring network activities. Our approach uses the fraction of idle channel time during the past history as an indication of local available bandwidth at a node. It may seem that this approach is too optimistic since it does not consider two points: first, some of the idle time can not be used due to the backoff algorithm in 802.11 ; second, when network load increases, the amount of bandwidth wasted in collision may increase. However, in [15], it is demonstrated that for 802.11, the amount of idle time and collision time is negligible even when the network is saturated. Therefore, using the fraction of idle channel time can be a simple and accurate estimator for available local bandwidth.

In general, the communication medium at a node can be perceived as being in four states: transmitting, receiving, noisy and idle. In the noisy state, a node senses a busy carrier but cannot interpret the content of the message. By monitoring the amount of time spent in these states, the local available bandwidth for a node can be estimated as follows:

$$
\begin{aligned}
\text { Total Time } & =\text { Transmitting Time }+ \text { Receiving Time }+ \text { Noisy Time }+ \text { Idle Time } \\
\text { Local Available Bandwidth } & =\frac{\text { Idle Time }}{\text { Total Time }} \times \text { Channel Capacity } \\
& =\left(1-\frac{\text { Transmitting Time }}{\text { Total Time }}-\frac{\text { Receiving Time }}{\text { Total Time }}-\frac{\text { Noisy Time }}{\text { Total Time }}\right) \times \text { Channel Capacity }
\end{aligned}
$$

To eliminate the effect of temporary variations in bandwidth calculations, the bandwidth estimation can be smoothed to reflect the long-term load on a node by using a weighted moving average.

\section{B. Contacting Relevant Neighbors}

As mentioned in Section II-A, nodes that are inside each other's carrier-sensing range may not be able to communicate directly if they are outside of each other's transmission range. However, since neighborhood available bandwidth is defined by the local available bandwidth of all neighboring nodes, calculating neighborhood available bandwidth requires actively exchanging bandwidth information with the nodes in carrier-sensing range. Since each node has a different view of the network, passively monitoring the network activities cannot provide information about local available bandwidth at other nodes. In CACP, we examine two approaches that can be used to coordinate the exchange of bandwidth information between neighboring nodes. 
In the first approach, a node broadcasts queries that have a limited hop count to attempt to reach all nodes in carrier-sensing range. This approach may not work in some topologies since a small hop count may not reach all nodes in carrier-sensing range and a large hop count may reach too many nodes. For example, in Figure 5, if Node A sends queries limited to 2 hops, Nodes G and E cannot be reached although they are in carrier-sensing range of Node A. By sending queries limited to 3 hops, Node H is falsely included although it is outside Node A's carrier-sensing range. Furthermore, although Node B is within carrier-sensing range of Node A, Node B cannot be reached by the queries no matter how many hops the queries take. The cost of this approach is based on the number of hops used to reach the neighbors. The first message is broadcast by the querying node; at each successive hop all neighbors rebroadcast the request. Since all of these transmissions are broadcast at the MAC layer, the amount of contention introduced by this approach may cause bad hot spots around the querying node and its n-hop neighbors.

The second approach takes advantage of the power control capabilities of today's wireless technologies. By allowing the sender to increase its transmission power to cover the entire carrier-sensing range, the sender can communicate with all nodes in its carrier-sensing range. The messages with increased power may contend with more nodes than a message at the normal power level, but to compensate, only one message is needed to reach all nodes in carrier-sensing range. It is important to note that this technique is only necessary to transfer bandwidth information. Data messages are sent at normal transmission power.

Evaluations of both of these approaches are presented in Section V-A and show that the power-control method imposes less overhead on the network when on average each node has more than three neighbors in transmission range. In most scenarios, the node density should be higher than this threshold to maintain connectivity in a network.

\section{Bandwidth Consumption}

The second challenge for CACP is to quantify the bandwidth that a new flow will consume so that it can be decided whether the available bandwidth can satisfy the flow's requirements. Because multiple nodes on the route of a flow may contend for the bandwidth at a single location and these contending nodes may not know about each other, estimating the bandwidth consumption of a flow requires not only the rate of flow but also the topology of the network.

For example in Figure 6, Flow X goes through path $A \rightarrow B \rightarrow C \rightarrow D \rightarrow E$. Since Nodes $\mathrm{A}, \mathrm{B}, \mathrm{C}, \mathrm{D}$ and $\mathrm{E}$ are in Node F's carrier-sensing range, they all contend for the bandwidth at Node F. If the rate of Flow X is $2 \mathrm{kbps}$, at least $10 \mathrm{kbps}$ bandwidth is consumed by Flow X at Node F. In general, assume Node $\mathrm{P}$ is in carrier-sensing range of N nodes on the route of an $\mathrm{R}$ bps flow. Each of the $\mathrm{N}$ nodes consumes $\mathrm{R}$ bps bandwidth at Node $\mathrm{P}$. If the flow goes through Node $\mathrm{P}$, the bandwidth consumption is $(R+N \times R)$ bps; if the flow does not go through Node $\mathrm{P}$, the bandwidth consumption is $(N \times R)$. We call the value $\mathrm{N}$ the contention count of the path.

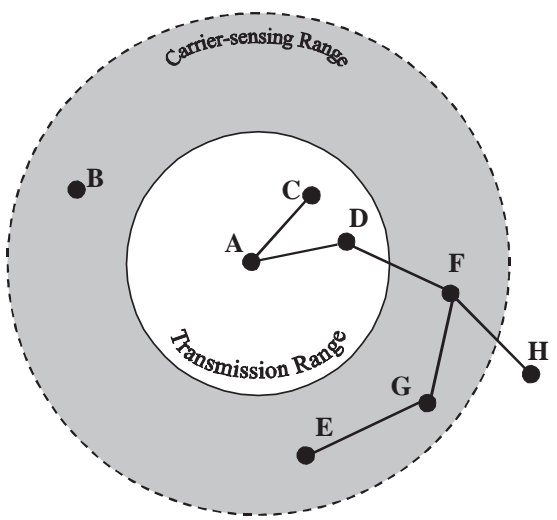

Figure 5. Multihop Approach

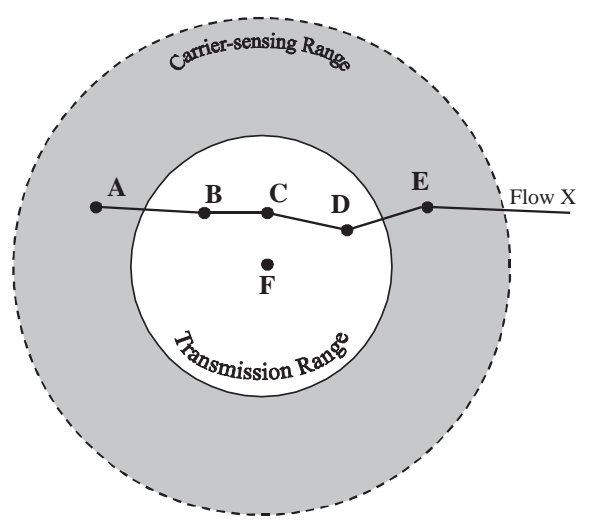

Figure 6. Bandwidth Consumption of a Flow

To determine the bandwidth consumption of a flow, every node could learn the up-to-date topology of the whole network so that the contention count and valid routes for a flow could be immediately calculated. However, this global approach is inappropriate for mobile environments, imposing heavy message overhead to avoid stale state information. In CACP, we use a local approach to reduce message overhead by monitoring the nodes in carrier-sensing range of the route of a flow. This optimization can be used because a flow cannot consume bandwidth at locations that are outside of carrier-sensing range of its route. Integrated with other parts of CACP, this local approach imposes no additional message overhead on the protocol. In Section IV-B, the local approach will be explained in detail. 


\section{PROTOCOL DESIGN}

CACP (Contention-aware Admission Control Protocol) works with single channel MAC layer protocols such as IEEE 802.11 [16], SEEDEX [7], etc. We use on-demand routing as the base for the design of CACP since it has low message overhead in the presence of mobility and can be naturally integrated with the local approach of bandwidth consumption estimation. CACP consists of two parts: route discovery and admission control. When a flow requests communication resources, the flow specifies its QoS requirements for communicating to the destination node. Route discovery and admission control are performed to find possible routes that may have enough bandwidth to meet these QoS requirements. Once a route has been found, the appropriate resources can be reserved on the chosen route.

\section{A. Route Discovery}

The aim of route discovery is to find a path between the sender and the receiver that has enough resources for the flow. CACP uses on-demand route discovery, similar to DSR [17], and performs admission control during the period of route discovery. When a source node has data to send, it initiates a route request by broadcasting a route request message to its neighbors. The route request message contains the address of the initiator of the request, the address of the destination and a record of the sequence of hops taken by the route request packet as it is propagated through the ad hoc network. We call this sequence of hops the partial path, which is used during the protocol to determine the contention count of a route and can also be used to eliminate circular routes. Each node that receives the route request message adds its own address to the partial path and rebroadcasts the message.

When the intended destination node receives a route request, the destination reverses the partial path in the request message and sends a route reply message back to the source along that path. The destination node sends one route reply for each route request it receives. Several reply messages may arrive at the source through different routes. The source chooses one of these paths to establish the connection and sends a connection request messages along the path indicated by the chosen reply message. When the destination receives the connection establishment message, a confirmation message is sent back to the source and communication can start.

\section{B. Distributed Admission Control Algorithm}

Route discovery will find possible routes to reach a destination. Admission control must be used to determine which of these routes can admit the new flow. At each node on the route, admission decisions must be based on the expected bandwidth consumption of the flow as well as the available bandwidth at the nodes and its neighbors in carrier-sensing range.

Local admission control is used to determine if sufficient local available bandwidth is available at a particular node. This decision must be based on the expected bandwidth consumption of the flow, which is determined by the contention count of the flow. To calculate the contention count, information about nodes in carrier-sensing range (including transmission range) is acquired by monitoring control messages. Information about these nodes is cached in what we call the active node set and removed after a period of inactivity to accommodate mobility in the network. Given the nodes in the route of the flow and the active node set, the contention count can be expressed as:

$$
\text { (Contention Count })=\mid(\text { Path }) \cap(\text { Active Node Set }) \mid \text {. }
$$

Neighborhood admission control is used to determine the impact of admission on all the nodes in carrier-sensing range by checking the neighborhood available bandwidth. Neighborhood admission control is achieved by broadcasting (via enhanced power or multihop techniques) an admission request message to all nodes in carrier-sensing range. Each node that receives the admission request performs local admission control for the flow. If local admission control fails, the node sends an admission rejection message to the initiator, and the neighborhood admission control fails.

During the route request phase, CACP uses purely local admission control as a first pass to cheaply eliminate routes without imposing extra message overhead. At this stage before a node rebroadcasts a route request message, the node makes a local admission control decision. The decision is based on partial information, since the whole route and so the final contention count of the route is not yet known. Also, since route request messages are sent with normal power, the nodes may only be aware of their one-hop neighbors by monitoring route request messages, leaving the active node set incomplete.

During the route reply phase, each node that receives the route reply message performs both local admission control and neighborhood admission control. Neighborhood admission control is used only in this phase for two reasons. First, neighborhood admission control is expensive since it requires querying neighboring nodes. Therefore, it is important to avoid using neighborhood admission control on nodes that are not along a viable route to the destination. These nodes are partly weeded out during the route request phase. Second, during the route request phase, only partial information is known. Therefore, neighborhood admission control would not be able to provide a final decision and would have to be performed later anyway. 


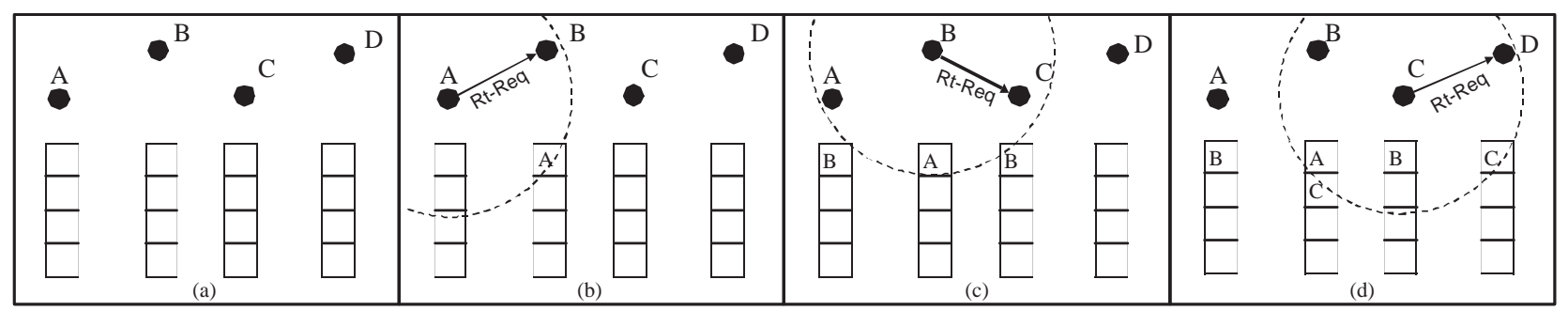

Figure 7. Route Request

In the route reply phase, since admission request messages are sent to reach the nodes in carrier-sensing range of the path, by monitoring the source of these messages, nodes can learn the part of the path that is in their carrier-sensing range and update their active node sets. Hence, accurate estimations of expected bandwidth consumption can be made in this phase. For example, assume on the route of a flow, there are $M$ nodes $N_{1}, N_{2}, \cdots N_{M}$ in carrier-sensing range of Node $X$. Suppose Node $X$ subsequently receives admission request messages from each of the $M$ nodes. When the last admission request message from Node $N_{M}$ reaches Node $X$, the active node set in Node $X$ includes the entire part of the route that is in its carrier-sensing range and the correct bandwidth consumption of the flow at Node $X$ can be calculated.

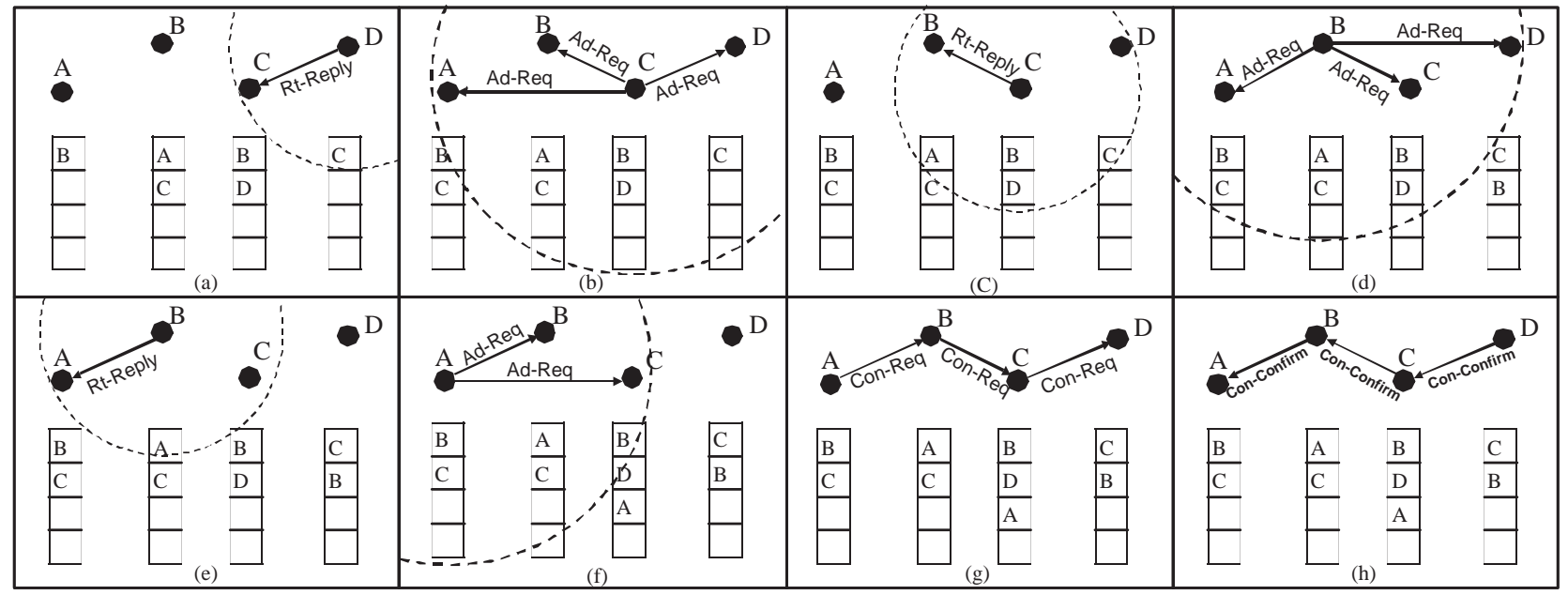

Figure 8. Successful Admission Control

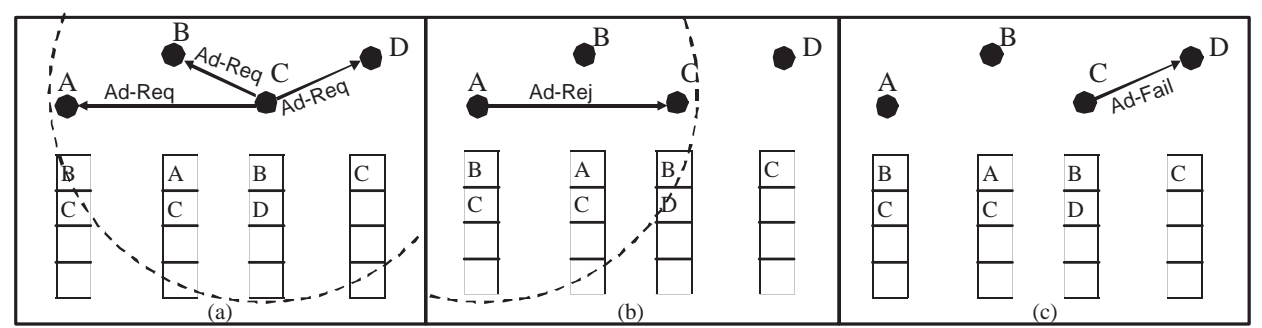

Figure 9. Admission Failure

To better illustrate CACP, we present an example of the process of route discovery and admission control in Figures 7,8 and 9 . The tables by the side of the nodes show the active node set of each node. Initially, no node has received any messages and the active node sets are empty (See Figure 7(a)).

As Node A initiates an X Mbps connection to node D, Node A invokes local admission control. If the flow passes, Node A broadcasts a route request message with partial path $\{A\}$ (See Figure 7(b)). When Node B receives the route request message, it adds Node $\mathrm{A}$ to its active node set, updates the partial path to $\{\mathrm{A}, \mathrm{B}\}$, and performs local admission control. The estimation 
of bandwidth consumption of the flow at this time can be expressed as:

$$
\begin{aligned}
\text { Estimated Bandwidth Consumption } & =(\text { Contention Count }(\mathrm{CC}) \text { of the Partial Path }) \times X+X \\
& =\mid(\text { Partial Path }) \cap(\text { Active Node Set }) \mid \times X+X \\
& =|\{A, B\} \cap\{A\}| \times X+X \\
& =2 X(\mathrm{bps})
\end{aligned}
$$

If Node B has enough local available bandwidth, Node B will rebroadcast the route request message with partial path $\{$ A,B $\}$ (See Figure 7(c)). Nodes $\mathrm{A}$ and $\mathrm{C}$ receive the message and update their active node sets. Node $\mathrm{C}$ performs local admission control and if the flow is admitted, Node $\mathrm{C}$ broadcasts the route request message with partial path $\{\mathrm{A}, \mathrm{B}, \mathrm{C}\}$ (See Figure $7(\mathrm{~d})$ ). When the destination $\mathrm{D}$ receives the route request message, it performs local admission control. If the flow passes the local admission control, Node D reverses the route and sends a route reply message back to Node C (See Figure 8(a)). When Node $\mathrm{C}$ receives the route reply, it performs neighborhood admission control by broadcasting an admission request message to all the neighbors in its carrier-sensing range as shown in Figure 9(a). Node A hears the message and adds Node $\mathrm{C}$ to its active node set. Node A knows that the bandwidth consumption of the flow will be $3 \mathrm{X}$ bps since $C C_{A}=\mid($ Partial Path $) \cap$ (Active Node Set) $|=|\{A, B, C, D\} \cap\{B, C\}|=|\{B, C\} \mid=2$ and Node A is on the route of the flow. If Node A's local available bandwidth cannot accommodate the flow, it sends an admission rejection message back to Node $\mathrm{C}$ and Node $\mathrm{C}$ then informs Node D about the failure of admission. (See Figure 9(a-c)).

If all of Node C's neighbors in carrier-sensing range have enough local available bandwidth, no node sends an admission rejection message. Node C forwards the route reply to Node B (See Figure 8(c)). Node B will perform neighborhood admission control in the same way as Node $\mathrm{C}$ and if the flow passes, Node B forwards the message to Node A. After the flow passes neighborhood admission control at Node $\mathrm{A}$, the route $A \rightarrow B \rightarrow C \rightarrow D$ is known to have enough bandwidth for the flow. Finally a connection request message is sent through the route to Node D and reservations are made along the route. When the connection confirmation message comes back, the data messages can start. This process is depicted in Figure 8(a-h).

\section{Resource Reservation}

Although the actual resource reservation policies and algorithms are beyond the scope of CACP, CACP provides several possible choices about when to make reservations. First, the sender can make resource reservations during the route request phase. This is very conservative and may result in the unnecessary reservation of resources along many failed routes. Second, it is also possible to make reservations during the reply phase. Such early reservation is some what less conservative, but resources may still be reserved and never used. However, reservations in the reply phase will reduce the chance of a connection being rejected during the connection establishment phase due to interference from other simultaneous connection requests. Finally, reservations can be made during the connection establishment phase, at which point the admission control protocol has approved the route.

\section{Mobility Management}

As mentioned in Section II-B, strict QoS can not be guaranteed in ad hoc network since the nodes of an ad hoc network are inherently subjected to mobility that is outside the network stack's ability to predict or control. Therefore, it is likely that QoS violations can be quite frequent in ad hoc networks. A possible method to reduce the frequency of QoS violations is to reserve some resources for unexpected events, such as when a new node moves into carrier-sensing range of a flow and contends for bandwidth with the flow. If some extra bandwidth is reserved to compensate for this, the QoS of the flow may still hold. However, a tradeoff must be made since the reserved bandwidth may not be used, reducing the efficiency of bandwidth usage in the network.

\section{Evaluation}

In this section, we evaluate CACP by mathematical analysis and simulations in ns2 [18]. In the simulations, we use IEEE 802.11 [16] as a MAC layer since it has almost no support for QoS. Therefore, the simulation results demonstrate the effectiveness of CACP rather than that of ad hoc QoS scheduling algorithms. By integrating QoS scheduling algorithms [7][8][9] with CACP, we expect the performance to be improved further.

First, we compare the effectiveness and the control-message overhead of using enhanced power and multihop approaches to contact neighbors in CACP. CACP-power sends admission request and admission rejection messages at enhanced power to reach nodes in carrier-sensing range. CACP-multihop sends these messages two hops to reach carrier-sensing range. Second, we present simulation results that demonstrate the success of CACP in a dynamic environment. Finally, we evaluate the overhead of CACP. 


\section{A. Overhead of Enhanced Power Approach and Multihop Approach}

Each time a control message is sent at an enhanced power level or in multihop mode, it causes more contention in its interference neighborhood than had it been sent as a normal message. We use the total number of times that nodes receive admission request and admission rejection messages as the measurement for the overhead of the two approaches. We first provide an analytical evaluation and then verify it through simulations.

\section{A.1. Analytical Study}

Suppose the transmission range of a node is $\mathrm{R}$ and the carrier-sensing range is $2 \mathrm{R}$. The total number of nodes in the network is a constant N. $\rho(x, y)$ is the density function, which represents the number of nodes in a unit area centered at location $(x, y)$. To simplify the problem, we assume that $\rho(x, y)$ in the carrier-sensing range is a constant. Assume an admission request message is generated at a location $(x, y)$. If CACP-multihop is used with 2 hops, which is most conservative in terms of overhead but may not reach al lnodes in carrier-sensing range, $\pi R^{2} \rho(x, y)$ nodes will hear the admission request message and each rebroadcasts the message to its own $\pi R^{2} \rho(x, y)$ neighbors. Therefore, the admission request message has been received $\pi R^{2} \rho(x, y)+\left(\pi R^{2} \rho(x, y)\right)^{2}$ times. If CACP-power is used, $4 \pi R^{2} \rho(x, y)$ nodes will hear the admission request message. Therefore, the ratio of the message overhead of CACP-Multihop vs. CACP-Power can be expressed as:

$$
\begin{aligned}
\text { OverheadRatio }(x, y) & =\frac{\text { MessageOverhead (CACP-Multihop, Location }(x, y))}{\text { MessageOverhead (CACP-Power, Location }(x, y))} \\
& =\frac{\pi R^{2} \rho(x, y)+\left(\pi R^{2} \rho(x, y)\right)^{2}}{4 \pi R^{2} \rho(x, y)} \\
& =\frac{1}{4}+\frac{\pi R^{2} \rho(x, y)}{4}
\end{aligned}
$$

According to Formula 1, at a certain location $(x, y)$, if the number of the neighbors in a node's transmission range, which is $\pi R^{2} \rho(x, y)$, exceeds 3, CACP-Multihop has a higher overhead than CACP-Power.

However, since the nodes in a network may not be evenly distributed, CACP-Power may have lower overhead in dense parts of the network than that of CACP-Multihop, while in sparse parts of the network, CACP-Multihop may have lower overhead. To compare the overall performance of the two approaches, we analyze the overhead ratio of the two approaches in a network where the nodes are not evenly distributed.

Let $\Delta_{x y}$ represent a very small square region centered at location $(x, y)$ with area $\Delta$. The node density in $\Delta_{x y}$ can be expressed as $\rho(x, y)$, and the number of nodes in $\Delta_{x y}$ can be expressed as $\rho(x, y) \Delta$. Assume that each node in the network has the same probability $q$ to generate an admission request. The expected number of initiators of admission requests in $\Delta_{x y}$ is therefore given by:

$$
E\left[\text { Number of Initiators in } \Delta_{x y}\right]=q \rho(x, y) \Delta
$$

If we divide the whole network area into square regions with area $\Delta$, the number of such regions can be written as:

$$
n=\frac{\text { Area of the network }}{\Delta}
$$

According to Formulas 1, 2 and 3, when the generation of an admission requests is random, the expected ratio of the overhead of the two approaches will be expressed as:

$$
\begin{aligned}
\text { OverheadRatio } & =\lim _{\Delta \rightarrow 0} \frac{\sum_{i=1}^{n}\left\{\pi R^{2} \rho\left(x_{i}, y_{i}\right)+\left[\pi R^{2} \rho\left(x_{i}, y_{i}\right)\right]^{2}\right\} q \rho\left(x_{i}, y_{i}\right) \Delta}{\sum_{i=1}^{n} 4 \pi R^{2} \rho\left(x_{i}, y_{i}\right) q \rho\left(x_{i}, y_{i}\right) \Delta} \\
& =\lim _{\Delta \rightarrow 0}\left[\frac{1}{4}+\frac{\pi R^{2}}{4} \frac{\sum_{i=1}^{n} \rho^{3}\left(x_{i}, y_{i}\right)}{\sum_{i=1}^{n} \rho^{2}\left(x_{i}, y_{i}\right)}\right] \\
& =\frac{1}{4}+\frac{\pi R^{2}}{4} \lim _{\Delta \rightarrow 0} \frac{\sum_{i=1}^{n} \rho^{3}\left(x_{i}, y_{i}\right)}{\sum_{i=1}^{n} \rho^{2}\left(x_{i}, y_{i}\right)} \quad, \text { where }\left(x_{i}, y_{i}\right) \text { is the coordination of the } i_{t h} \text { small region with area } \Delta
\end{aligned}
$$

According to general means inequality, for $n$ positive numbers $X_{1}, X_{2}, \cdots X_{n}$,

$$
\begin{aligned}
\left(\frac{1}{n} \sum_{i=1}^{n} X_{i}^{2}\right)^{\frac{1}{2}} \leq\left(\frac{1}{n} \sum_{i=1}^{n} X_{i}^{3}\right)^{\frac{1}{3}} & \Longrightarrow\left(\sum_{i=1}^{n} X_{i}^{2}\right)\left(\frac{1}{n} \sum_{i=1}^{n} X_{i}^{2}\right)^{\frac{1}{2}} \leq \sum_{i=1}^{n} X_{i}^{3} \\
& \Longrightarrow \frac{\sum_{i=1}^{n} X_{i}^{3}}{\sum_{i=1}^{n} X_{i}^{2}} \geq\left(\frac{1}{n} \sum_{i=1}^{n} X_{i}^{2}\right)^{\frac{1}{2}} \geq \frac{1}{n} \sum_{i=1}^{n} X_{i}
\end{aligned}
$$




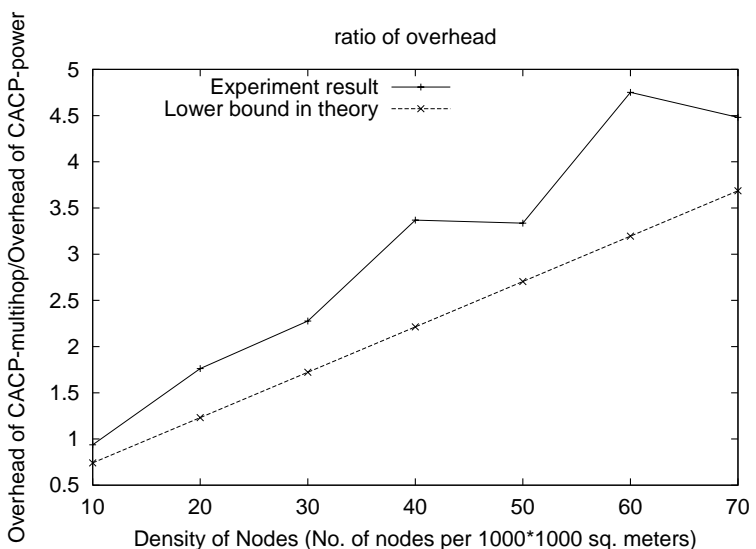

Figure 10. Overhead Ratio of CACP-multihop vs. CACP-power

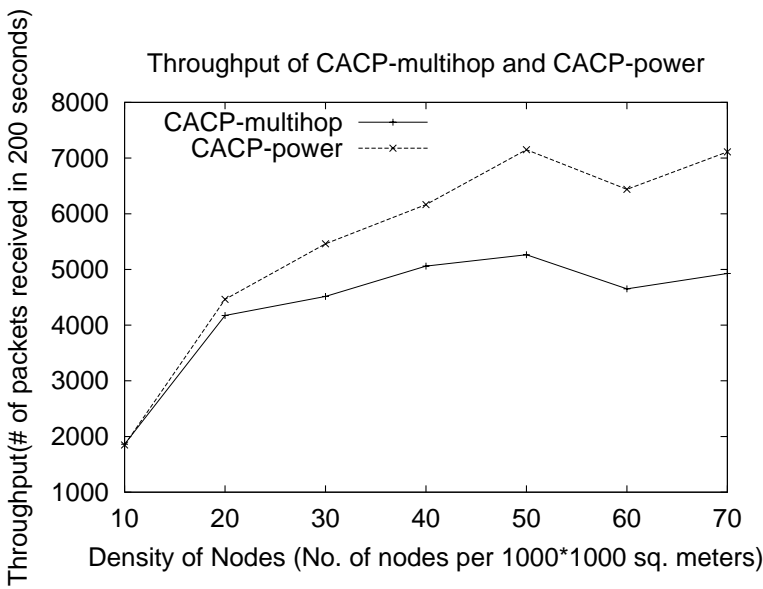

Figure 11. Throughput of CACP-multihop and CACP-power

The equality holds when $X_{1}=X_{2}=\cdots=X_{n}$. Therefore, we have:

$$
\frac{\sum_{i=1}^{n} X_{i}^{3}}{\sum_{i=1}^{n} X_{i}^{2}} \text { is minimized when } X_{1}=X_{2}=\cdots=X_{n} .
$$

If the number of nodes in the network is a constant N, according to Formula 3 ,

$$
\lim _{\Delta \rightarrow 0} \sum_{i=1}^{n} \rho\left(x_{i}, y_{i}\right) \Delta=N \Longrightarrow \lim _{\Delta \rightarrow 0} \frac{\sum_{i=1}^{n} \rho\left(x_{i}, y_{i}\right) \Delta}{n \Delta}=\frac{N}{\text { Area of the network }} \Longrightarrow \lim _{\Delta \rightarrow 0} \frac{\sum_{i=1}^{n} \rho\left(x_{i}, y_{i}\right)}{n}=\frac{N}{\text { Area of the network }}
$$

According to Formulas 4, 5 and 6, we have

$$
\begin{aligned}
\text { OverheadRatio } & =\frac{1}{4}+\frac{\pi R^{2}}{4} \lim _{\Delta \rightarrow 0} \frac{\sum_{i=1}^{n} \rho^{3}\left(x_{i}, y_{i}\right)}{\sum_{i=1}^{n} \rho^{2}\left(x_{i}, y_{i}\right)} \\
& \geq \frac{1}{4}+\frac{\pi R^{2}}{4} \lim _{\Delta \rightarrow 0}\left[\frac{1}{n} \sum_{i=1}^{n} \rho\left(x_{i}, y_{i}\right)\right] \\
& =\frac{1}{4}+\frac{\pi R^{2}}{4} \frac{N}{\text { Area of the network }}
\end{aligned}
$$

The equality holds when $\rho(x, y)$ is a constant for all $(x, y)$ and the theoretical lower bound of the overhead ratio of CACPmultihop vs. CACP-power will be $\left(\frac{1}{4}+\frac{\pi R^{2}}{4} \rho\right)$, which can be achieved when the density of nodes in the network is a constant $\rho$. Therefore, the higher the density of the network, the lower the overhead of CACP-power compared with CACP-multihop

\section{A.2. Simulation and Results}

Several scenarios were tested to verify the result of the above analysis under different node densities. In the simulations, mobile hosts are randomly distributed in $1000 \times 1000$ square meters. The number of nodes ranges from 10 to 70 . Five scenarios are examined for each density. Radio transmission range is 250 meters and carrier-sensing range is 550 meters. The bandwidth of the channel is 2 Mbps. Pairs of Nodes are randomly chosen to establish connections with a CBR traffic source. The maximum number of connections is 10. The CBR traffic generates 10512 Bytes packets/second. The movement pattern of nodes is the random waypoint model [19]. The speed of nodes is 5 meters/second and the pause time is 20 seconds. The simulations run for 200 seconds.

Figure 10 shows the overhead ratio of CACP-multihop vs. CACP-power from the simulations and the theoretical analysis. As expected, the overhead ratio from the simulations is higher than the lower bound calculated. This is because, in the simulations, the nodes are randomly distributed in the network and may cluster in some parts of the network. The simulations support that CACP-power has a lower overhead than CACP-multihop when the average density of nodes is more than 15.3 nodes $/ 10^{6} \mathrm{~m}^{2}$, which also means that on average, there are three neighbors in a node's transmission range. This density threshold is very low for maintaining connectivity in an ad hoc network, as shown in Figure 11. When the node density is small, the throughput under both approaches is low because of the low connectivity. As the density of nodes increases, the throughput of CACP-power becomes larger than that of CACP-multihop due to its low message overhead. Therefore, we use CACP-power in the remaining simulations to examine the performance of CACP. 


\section{B. Bandwidth Management}

In this section, we examine the effectiveness of CACP's admission control in a random topology when mobility is present. To our knowledge, no protocol considers the resources of nodes in carrier-sensing range when performing admission control. Therefore, we use DSR as a baseline for our on-demand approach for evaluating both flow characteristics and protocol message overhead. Our simulations show that without careful bandwidth allocation, the ad hoc network can easily be overwhelmed by too much traffic. With the help of CACP, however, bandwidth is reserved for flows according to the bandwidth requirements of the flows and the current network capacity.

In these simulations, there are 50 mobile hosts randomly distributed in $1000 \times 1000$ square meters. 10 randomly chosen pairs of nodes try to establish a connection with each other with a CBR traffic source generating $80 \mathrm{kbps}$ and transmit the data at 20 packets/second. The movement pattern of nodes is the random waypoint model. The speed of nodes is 5 meters/second and the pause time is 20 seconds. The simulation runs for 200 seconds.

For IEEE 802.11, admission control cannot assume that all the channel capacity can be reserved for flows. This is because many control messages, such as route request messages and QoS management messages, are not included in per flow resource reservations and there is no scheduling algorithm in IEEE 802.11 that prevents these control messages from contending with ordinary packet transmission. Therefore, CACP must reserve some bandwidth to accommodate future control messages. Hence, Bandwidth Utilization, which is defined as Used Bandwidth/Total Channel Bandwidth, should be controlled by CACP so that it does not go beyond a certain threshold named Bandwidth Utilization Threshold. In our simulations, CACP refuses the admission request of a flow when the new flow would increase the expected Bandwidth Utilization over the Bandwidth Utilization Threshold. This expected Bandwidth Utilization can be calculated as follows:

$$
\begin{aligned}
\text { Expected Bandwidth Utilization } & =\frac{\text { Expected Used Bandwidth }}{\text { Total Channel Bandwidth }}=1-\frac{\text { Expected Free Bandwidth }}{\text { Total Channel Bandwidth }} \\
& =1-\frac{\text { The Neighborhood Available Bandwidth - Bandwidth Consumption of the New Flow }}{\text { Total Channel Bandwidth }}
\end{aligned}
$$

To evaluate the effect of the Bandwidth Utilization Threshold, 75 simulations are examined with Bandwidth Utilization Thresholds ranging from $30 \%$ to $100 \%$. A network operator can use the following results to determine the appropriate value of Bandwidth Utilization Threshold for a desired QoS.

In Figure 12(a) and Figure 12(b), we compare the average delay of packets and the average rate of flows in the last 50 seconds of the simulations of DSR and CACP-power since at this time all traffic sources in the simulation have started. By using 50\% Bandwidth Utilization Threshold, CACP-power ensures that the average delay of flows is less than $8 \mathrm{~ms}$ and the throughput of each flow is 20 packets/second. With 50\% to 75\% Bandwidth Utilization Threshold, the network shows larger delays in the range of $15 \mathrm{~ms}$ to $34 \mathrm{~ms}$ and lower throughput for each flow in the range of 19.5 packets/second to 18 packets/second. When bandwidth utilization is high (between $75 \%$ to $100 \%$ ), average packet delay is as high as $70 \mathrm{~ms}$ to 150 ms and throughput drops from 17.5 packets/seconds to 14 packets/seconds. At the same time, average packet delay for DSR is $220 \mathrm{~ms}$ and throughput is 13.8 packets/seconds, which is worse than that of CACP.

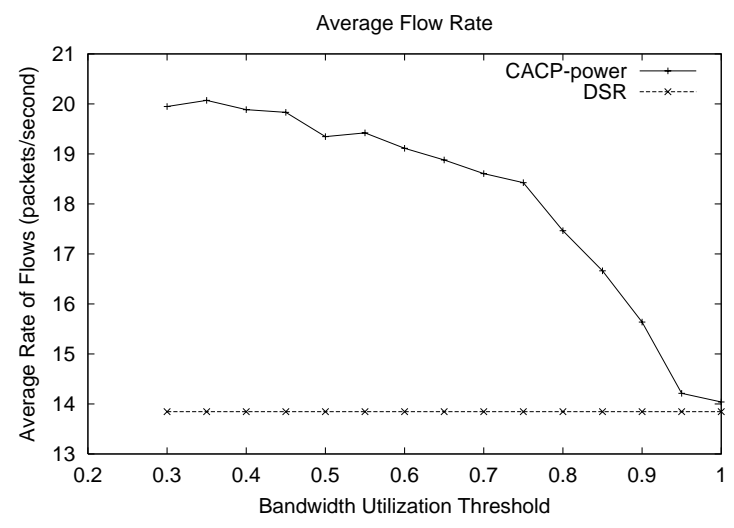

(a) Average Flow Rate

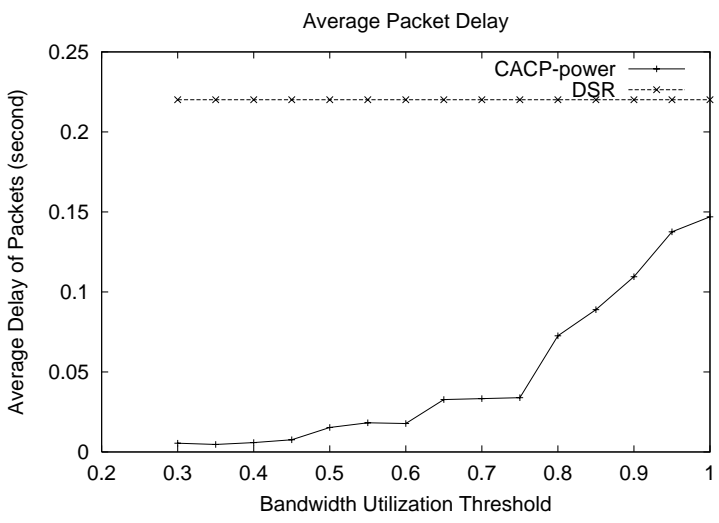

(b) Average Packet Delay

Figure 12. Performance under Different Bandwidth Utilization Thresholds

The bad performance of DSR comparing to CACP during the last 50 seconds is due to the fact that DSR imposes no control on the amount of traffic in the network. To better illustrate how CACP controls the admission of flows, traces of the flows 


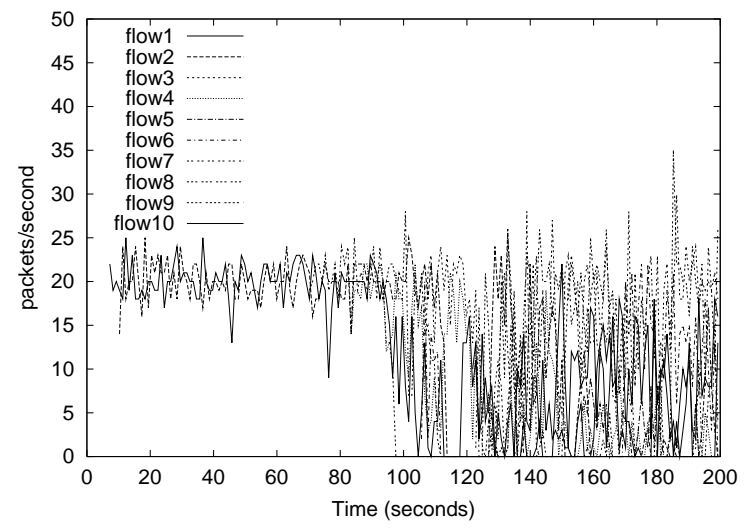

(a) All ten flows

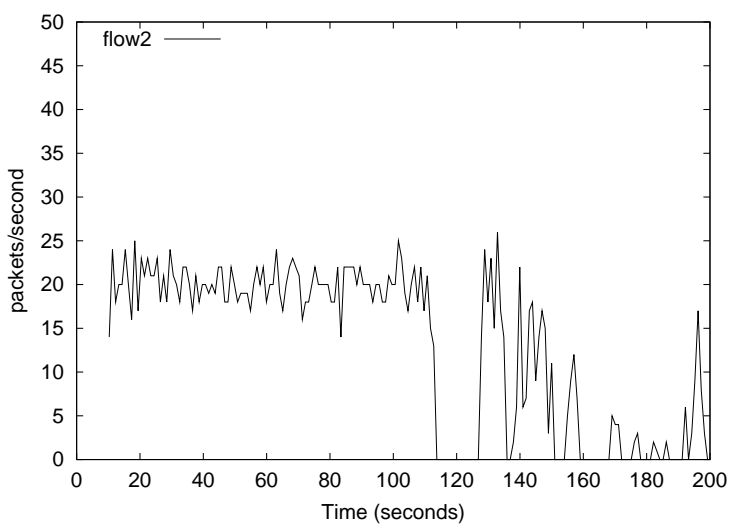

(b) Flow 2

Figure 13. Throughput of the flows in DSR

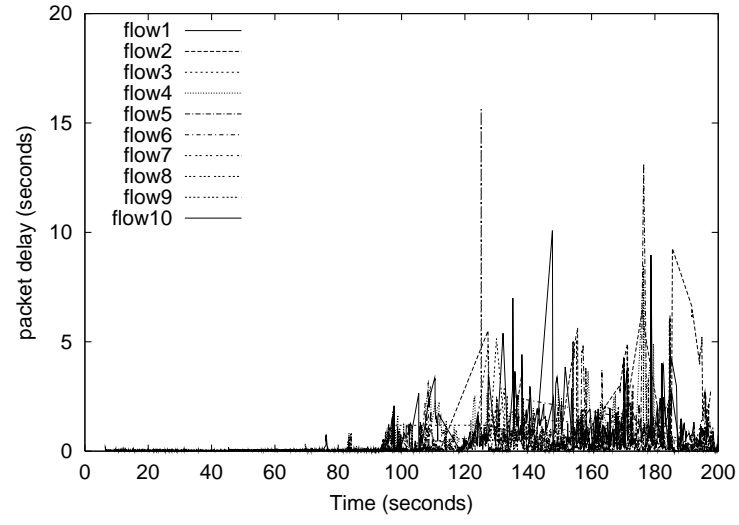

(a) All ten flows

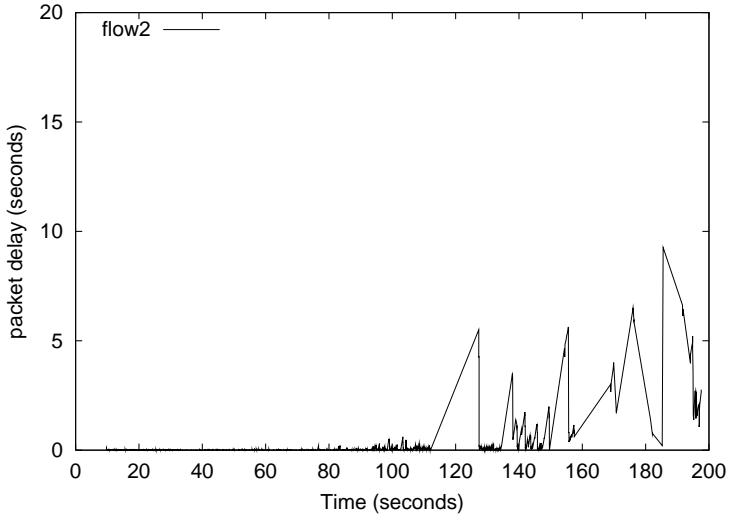

(b) Flow 2

Figure 14. Packet delay of the flows in DSR

in one of the above simulations are shown in Figures 15 and 16. The Bandwidth Utilization Threshold in this simulation is 50\%. For comparison, traces of the flows in DSR are also shown in Figures 13 and 14. Figure 13(a) and Figure 15(a) show the throughput of each flow in terms of the number of network data packets received per second in DSR and CACP, while Figure 14(a) and Figure 16(a) show the delay. (Note that the scale of y-axis in Figure 16 is 20 times smaller than that in Figure 14.) Figures 13(b), 14(b), 15(b) and 16(b) pick Flow 2 from the ten flows and show its throughput and packet delay. Because there is no admission control for DSR, as the number of flows injected in the network increases and finally passes the capacity of the network at about 125 seconds, all flows in the network experience reduced and dramatically varying throughput and large packet delays. On the other hand, CACP can successfully guarantee the performance of the network by controlling the traffic load. Eight out of the ten flows are admitted by CACP. The other flows are rejected due to lack of bandwidth. Variations in delay and flow rate can happen since the lower layer IEEE 802.11 does not have any scheduling support. Even in this situation, for many QoS applications that use buffers to accommodate changes in bandwidth and delay, the relatively short variations in bandwidth and delay will not be a problem. For applications with stricter QoS requirement, QoS aware MAC layer protocols in [7][8][9] can be used with CACP. However, we are still investigating this combination of CACP with such protocols.

\section{Admission Control Message Overhead}

The main message overhead of CACP compared to DSR is the admission request and admission rejection messages. By comparing the number of admission request and admission rejection messages with the route request messages, we demonstrate that the admission control message overhead of CACP is acceptable. 


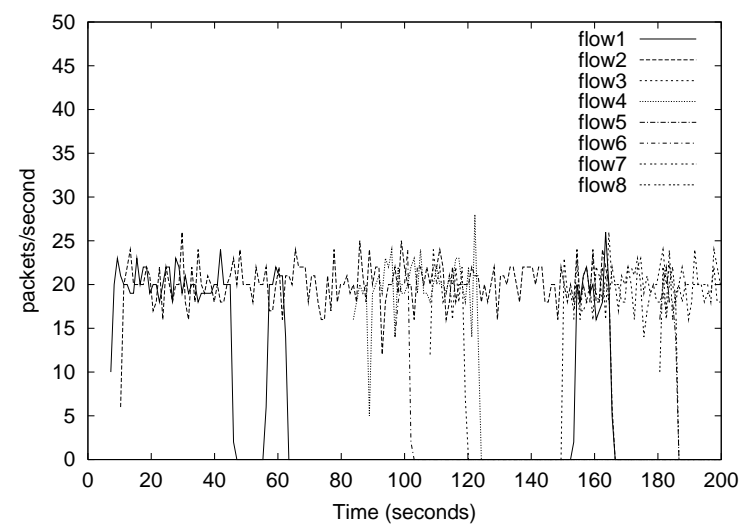

(a) All admitted flows

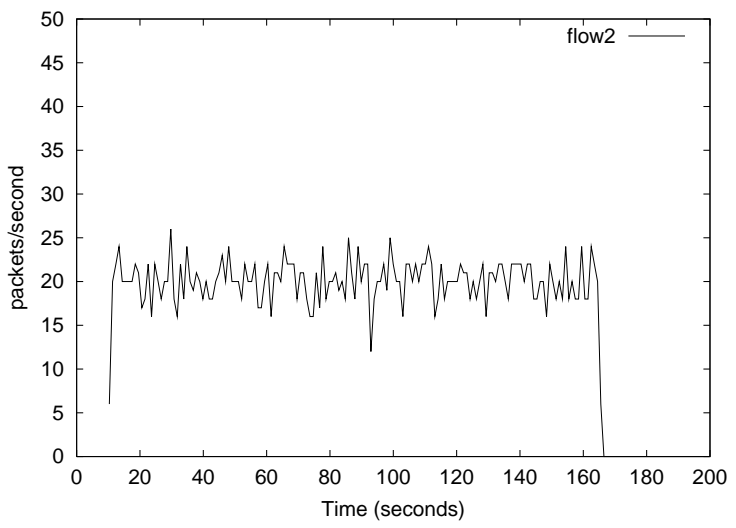

(b) Flow 2

Figure 15. Throughput of the flows in CACP with $50 \%$ bandwidth utilization

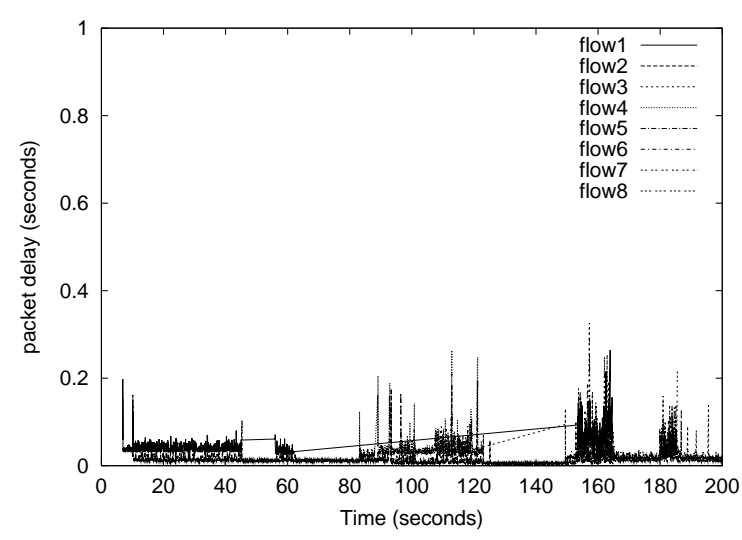

(a) All admitted flows

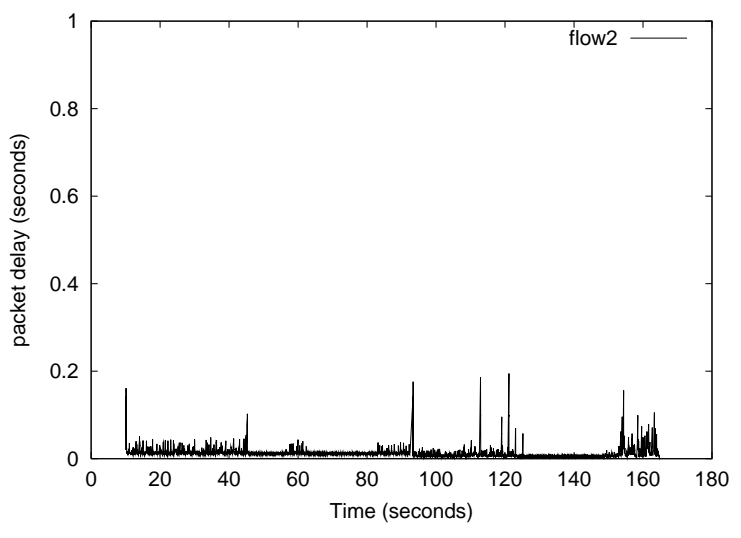

(b) Flow 2

Figure 16. Packet delay of flows in CACP with 50\% bandwidth utilization

The number of admission request, admission rejection and route request messages are recorded in all the previous simulations. In the simulations described in Section V-B, where the performance of CACP under different bandwidth utilization is examined, the number of admission request, admission rejection and route request messages is shown in Figure 17(a). In the simulations in Section V-A, where we compare the overhead of CACP-power to that of CACP-multihop in respect to different node density, the number of admission request, admission rejection and route request messages for CACP-power is recorded and shown in Figure 17(b). Although there is certainly overhead to perform admission control, it only represents a fractional increase beyond the overhead from route request messages which are unavoidable. We believe that this is acceptable, given the benefit of admission control.

\section{CONCLUSION}

We have presented a contention-aware admission control protocol based on a single channel medium. The main contribution of our research is the inclusion of information from nodes inside carrier-sensing range and outside transmission range of the node performing the admission control. The success of our protocol is shown through simulations, where CACP effectively manages requests for bandwidth beyond the capabilities of the network with acceptable overhead. Since CACP is only the admission control part of a QoS routing protocol, we are currently evaluating the use of CACP upon various QoS aware MAC protocols.

\section{REFERENCES}

[1] D. Maltz, "Resource management in multi-hop ad hoc networks," in Technical Report CMU CS 00-150, School of Computer Science, Carnegie Mellon University, July 2000. 


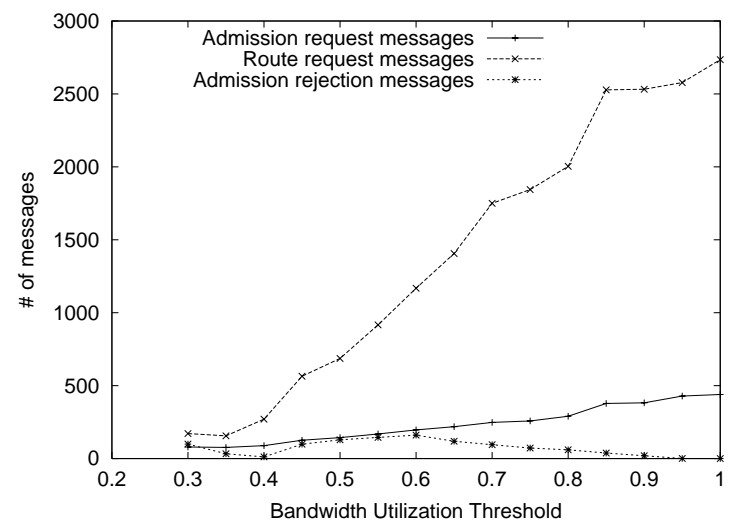

(a) Overhead vs. Bandwidth Utilization Threshold

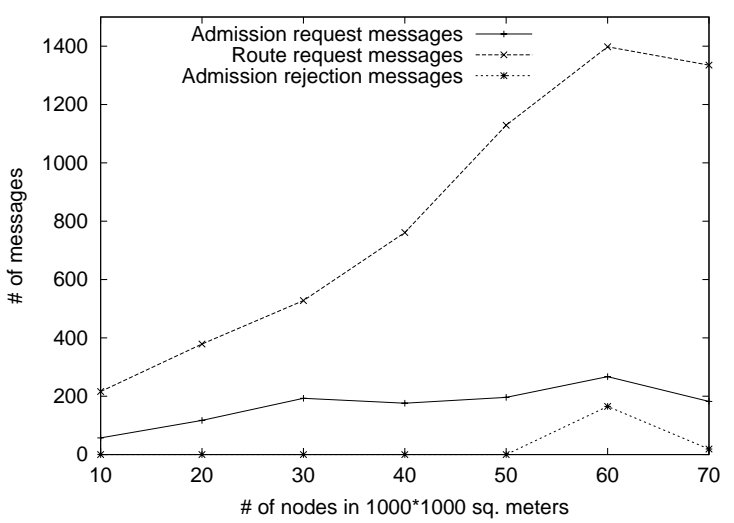

(b) Overhead vs. Node Density

Figure 17. Overhead of Route Request and Admission Request Messages

[2] Y.-C. Hsu and T.-C. Tsai, "Bandwidth Routing in Multihop Packet Radio Environment," in Proceedings of the 3rd International Mobile Computing Workshop, 1997.

[3] T.-W. Chen, J. T. Tsai, and M. Gerla, "QoS Routing Performance in Multihop Multimedia Wireless Networks," in Proceedings of IEEE International Conference on Universal Personal Communications (ICUPC’97), 1997.

[4] C.R. Lin and Chung-Ching Liu, "An On-demand QoS Routing Protocol for Mobile Ad Hoc Networks," in IEEE Global Telecommunications Conference (GLOBECOM'O0), 2000.

[5] Chenxi Zhu and M. Scott Corson, "QoS Routing for Mobile Ad Hoc Networks," Tech. Report CSHCN TR 2001-18, Institute for system research, University of Maryland, 2001.

[6] C. R. Lin and J.-S. Liu, "QoS Routing in Ad Hoc Wireless Networks," IEEE Journal on Selected Areas in Communications, vol. 17, no. 8, pp. 1426-1438, Nov./Dec. 1999.

[7] R. Rozovsky and P. R. Kumar, "SEEDEX: A MAC protocol for ad hoc network," in Proceedings of The ACM Symposium on Mobile Ad Hoc Networking \& Computing, Long Beach, 2001.

[8] V. Kanodia, C. Li, A. Sabharwal, B. Sadeghi, and E. Knightly, "Distributed Multi-Hop Scheduling and Medium Access with Delay and Throughput Constraints," in Proceedings of the seventh annual international conference on Mobile computing and networking (MobiCom'01), Rome, Italy, 2001.

[9] Haiyun Luo, Songwu Lu, Vaduvur Bharghavan, Jerry Cheng, and Gary Zhong, "A Packet Scheduling Approach to QoS support in Multihop Wireless Networks," ACM Journal of MObile Networks and Applications(MONET), Special Issue on QoS in Heterogeneous Wireless Networks, 2002.

[10] Gahng-Seop Ahn, Andrew Campbell, Andras Veres, and Li-Hsiang Sun, "SWAN: Service Differentiation in Stateless Wireless Ad Hoc Networks," in Proceedings of Infocom'02, New York, 2002.

[11] Seoung-Bum Lee, Gahng-Seop Ahn, Xiaowei Zhang, and Andrew Campbell, "INSIGNIA: An IP-Based Quality of Service Framework for Mobile Ad Hoc Networks," Journal of Parallel and Distributed computing, Special issue on Wireless and Mobile Computing and Communications, vol. 60, pp. $374-406,2000$

[12] Michael G. Barry, Andrew T. Campbell, and Andras Veres, "Distributed Control Algorithms for Service Differentiation in Wireless Packet Networks," in Proceedings of Infocom'01, 2001.

[13] Ram Ramanathan and Martha Steenstrup, "Hierarchically-organized, multihop mobile wireless networks for quality-of-service support," Mobile Networks and Applications, vol. 3, no. 1, pp. 101-119, 1998.

[14] Shree Murthy and J. J. Garcia-Luna-Aceves, "A routing architecture for mobile integrated services networks," Mobile Networks and Applications, vol. 3, no. 4, pp. 391-407, 1999.

[15] Giuseppe Bianchi, "Performance analysis of the IEEE 802.11 distributed coordination function," IEEE Journal on Selected Areas in Communications, vol. 18, no. 3, 2000 .

[16] IEEE Computer Society, "802.11: Wireless LAN Medium Access Control (MAC) and Physical Layer (PHY) Specifications," .

[17] David Johnson and David Maltz, Dynamic source routing in ad hoc wireless networks, in Mobile Computing, Kluwer Academic Publishers, 1996.

[18] Kevin Fall and Kannan Varadhan, "NS notes and documentation," in The VINT Project, UC Berkely, LBL, USC/ISI, and Xerox PARC, 1997.

[19] J.Broch, D.A.Maltz, D.B.Johnson, Y.-C.Hu, and J.Jetcheva, "A performance comparison of multihop wireless ad hoc network routing protocol," in ACM Mobicom'98, 1998. 